\title{
The content of endogenous hormones and sugars in the process of early somatic embryogenesis in the tree fern Cyathea delgadii Sternb.
}

\author{
Małgorzata Grzyb $^{1} \cdot$ Agnieszka Kalandyk $^{2} \cdot$ Piotr Waligórski $^{2} \cdot$ Anna Mikuła $^{1}$
}

Received: 30 November 2016 / Accepted: 31 January 2017 / Published online: 15 February 2017

(C) The Author(s) 2017. This article is published with open access at Springerlink.com

\begin{abstract}
Somatic embryogenesis (SE) of Cyathea delgadii presents a model system for investigating the mechanisms associated with the acquisition of embryogenic competence by single epidermal cells of stipe explants cultured on plant growth regulator-free medium. The present work reveals relationship between endogenous hormone and sugar content in the process of early SE in $C$. delgadii. By comparing two types of initial explants, i.e. incapable (nonetiolated) and capable (etiolated) of SE, it was established that in etiolated explants, the glucose, fructose, sucrose, and abscisic acid (ABA) contents diminished, but indole3-acetic acid (IAA) and cytokinins (CKs; i.e. cis/trans zeatin, cis/trans-zeatin riboside, kinetin, kinetin riboside, isopentenyladenosine) contents increased. The ratios between phytohormones revealed that a high concentration of $\mathrm{ABA}$ is the main factor inhibiting SE induction. Because
\end{abstract}

Electronic supplementary material The online version of this article (doi:10.1007/s11240-017-1185-8) contains supplementary material, which is available to authorized users.

Małgorzata Grzyb

mgrzyb@obpan.pl

Agnieszka Kalandyk

a.kalandyk@ifr-pan.edu.pl

Piotr Waligórski

pewalig7@gmail.com

Anna Mikuła

amikula@obpan.pl

1 Polish Academy of Sciences Botanical Garden-Center for Biological Diversity Conservation in Powsin, Prawdziwka 2, 02-973 Warsaw, Poland

2 The Franciszek Górski Institute of Plant Physiology, Polish Academy of Sciences, Niezapominajek 21, 30-239 Cracow, Poland of explant excision, a dramatic reduction in concentration of all phytohormones studied was observed, but hormonal balance and sugar content remained almost unchanged. During the 14-day-long culture, the ABA/CKs and ABA/ IAA ratios remained constant, whereas the greatest differences were detected for the IAA/CKs and Z-type/iPA cytokinin ratios. Excluding day 6 of culture, cytokinins were found to be the predominant phytohormones over IAA. An almost 12-fold increase in soluble sucrose concentration at day 6 of culture might be the switch to the SE expression phase. Frequent cell divisions leading to somatic embryo formation are clearly associated with increase in trans-zeatin riboside content.

Keywords Etiolation - Explant excision · Highperformance liquid chromatography · PGR-free medium . Phytohormones

$\begin{array}{ll}\text { Abbreviations } \\ \text { ABA } & \text { Abscisic acid } \\ \text { c/t-Z } & \text { Cis/trans zeatin } \\ \text { c/t-ZR } & \text { Cis/trans-zeatin riboside } \\ \text { CKs } & \text { Total concentration of cytokinins } \\ \text { FW } & \text { Fresh weight } \\ \text { HPLC } & \text { High-performance liquid chromatography } \\ \text { IAA } & \text { Indole-3-acetic acid } \\ \text { iPA } & \text { Isopentenyladenosine } \\ \text { Kin } & \text { Kinetin } \\ \text { KinR } & \text { Kinetin riboside } \\ \text { PGR } & \text { Plant growth regulator } \\ \text { SE } & \text { Somatic embryogenesis }\end{array}$




\section{Introduction}

Somatic embryogenesis (SE) is an important pathway for the regeneration of plants, as well as an attractive system for the study of the morphology, physiology, and genetic mechanisms of embryo induction and development (Elhiti et al. 2013; Fehér 2015). Although this process was first described many years ago, our present knowledge about the acquisition of embryogenic competence by somatic cells remains unknown, and it is unexplored in ferns.

Understanding of the physiological mechanism that underlies the induction of SE is essential for the regulation of this process. Phytohormones are employed as signalling molecules for the induction of SE by mediating the signal transduction cascade leading to the reprogramming of gene expression patterns (Elhiti et al. 2013). Some hormones, such as abscisic acid (ABA) and cytokinins have been shown to help regulate the sugar metabolism and transport. On the other hand, sugars regulate the synthesis, conjugation and transport of phytohormones (for example $\mathrm{ABA}$ ), and also the expression of components of phytohormoneresponse pathways (Gibson 2004). A direct link between sugar signalling and hormone biosynthesis has been described by Eveland and Jackson (2011). At present, it is not surprising that the availability of sugars in the induction medium helps regulate developmental processes in ferns (Mikuła et al. 2015a). However, the changes in endogenous sugar contents during SE induction have not yet been studied.

The relationships between endogenous hormone contents and SE have been the subject of many studies. However, most of them are limited to the comparisons of the non-embryogenic and embryogenic callus (Jiménez and Bangerth 2000; Pintos et al. 2002; Jiménez et al. 2005), the non-embryogenic explants and those with somatic embryos developed on the explant surface (Pérez-Jiménez et al. 2013), or the genotypes/explants with different embryogenic capability (Wenck et al. 1988; Centeno et al. 1997; Limanton-Grevet et al. 2000; Jiménez and Bangerth 2001a). Only a few reports on the progressive hormonal changes that occur during SE are available, but they differ in the type of explant, method (plant growth regulators or other stress factors) and manner of somatic embryo induction (i.e. direct or indirect), and culture conditions (photoperiodic or darkness) employed (Grieb et al. 1997; Charrière et al. 1999; Jiménez and Bangerth 2001b; Yang et al. 2012; Ayil-Gutiérrez et al. 2013). In these studies, the dynamic changes in the content of indole-3-acetic acid (IAA) (Ayil-Gutiérrez et al. 2013), or IAA and ABA and cytokinins (Grieb et al. 1997; Charrière et al. 1999), and also in gibberellin concentrations (Jiménez and Bangerth 2001a) during the induction of SE were described for Coffea canephora Pierre ex Froehner, Daucus carota
L., Helianthus annuus L., and Zea mays L., respectively. Because the ratio of endogenous hormones controls a mode of morphogenetic response, the ratio of IAA or ABA to cytokinins (Żur et al. 2015), or the ratio of ABA to IAA (Centeno et al. 1997), or of various types of cytokinins (e.g. zeatin (Z)-type and isopentenyl (iP)-type; Grieb et al. 1997; Pintos et al. 2002) tend to be good indicators of the embryogenic competence of explants.

In plant material, endogenous hormone contents may be affected by many factors. For example, exogenously applied plant growth regulators (PGRs) can interact with phytohormones and change their concentrations (AyilGutiérrez et al. 2013). Another factor causing hormonal balance disorders is explant excision (Thornburg and $\mathrm{Li}$ 1991; Yang et al. 2012). It was showed that wounding is the first event that provides signals for triggering the entire regenerative process (Iwase et al. 2011; Chen et al. 2016). Although excision is a commonly used procedure for the introduction of explants into in vitro culture, little attention has been paid to this treatment so far. Phytohormone concentrations and their signalling pathways can also be regulated by changes in the photoperiodic conditions (Symons and Reid 2003; Pacholczak et al. 2005). As a result of the light deficit, plants show a dramatic elongation of the stem (Suzuki and Kerbauy 2006). The plant response is often linked to reduced leaf development, enhanced apical dominance, and a re-allocation of resources from storagefavouring shoot elongation to reproductive growth (Suzuki and Kerbauy 2006; Mikuła et al. 2015b). Dark treatment of donor plantlets was reported to favour SE in Helianthus annuus L. (Fiore et al. 1997), Dendranthema grandiflora Tzvelev. (May and Trigiano 1991) and Cyclamen persicum Mill. (Takamura and Tanaka 1996). Moreover, Pacholczak et al. (2005) discovered a correlation between light intensity and total soluble sugars and endogenous ABA and IAA contents.

By culturing stipe explants of the tree fern Cyathea delgadii Sternb. on PGR-free half-strength Murashige and Skoog's (1/2MS) medium, in darkness, we established a unique model system for the study of SE (Mikuła et al. 2015b). In this system, somatic embryos regenerate directly from the single epidermal cells of stipe explants. However, the capacity of $C$. delgadii explants to undergo SE depends on the prevailing photoperiodic conditions during the development of plantlets (Mikuła et al. 2015a). Explants excised from sporophytes that developed under 16/8 photoperiod conditions are incapable of SE. In contrast, in etiolated explants, the first cell divisions typically starts at 10th day of culture, and during the next 4-6 days, many somatic embryos at linear developmental stage are observed (Mikuła et al. 2015b). The present study was undertaken to find out if the etiolation, which is a key factor in the capacity of stipe explants to form somatic embryos, has an effect 
on the content and balance of hormones and sugars. The study was also designed to determine possible relationships between the concentration and balance of above-mentioned compounds in etiolated explants during their 14-day-long culture on PGR-free medium.

\section{Materials and methods}

\section{Plant material and culture initiation}

Embryogenic culture of $C$. delgadii was established following the procedure described earlier by Mikuła et al. (2015b). The somatic embryo-derived sporophytes were cultured on PGR-free medium containing half-strength Murashige and Skoog's (Murashige and Skoog 1962) macro- and micro-nutrients and full complement of vitamins (1/2MS) and 2\% (w/v) sucrose, solidified with $0.7 \%$ plant agar (Duchefa Biochemie); pH 5.8. The cultures were maintained under the photoperiodic conditions $(16 / 8$ light-dark regime; $50 \mu \mathrm{M} \mathrm{m}^{-2} \mathrm{~s}^{-1}$; defined as non-etiolated) or in constant darkness (defined as etiolated) for 5 months.

In experiments described here, sporophytes that had developed 4 or 5 leaves were used as a source of explants. The stipe explants measuring $2.5 \mathrm{~mm}$ in length were excised from the first frond of non-etiolated and etiolated sporophytes (Mikuła et al. 2015a) and used for high-performance liquid chromatography (HPLC) analysis. Moreover, etiolated explants were cultured on $1 / 2 \mathrm{MS}$ induction medium supplemented with $1 \%(\mathrm{w} / \mathrm{v})$ sucrose, in constant darkness for 14 days. The plant material was collected for HPLC analysis every 2 days.

\section{Samples preparation for HPLC analysis}

Excised explants were immediately frozen in liquid nitrogen. The plant samples (about 80 explants per sample; $35 \mathrm{mg}$ of FW) were lyophilised and homogenised in an icecold mixture of methanol, water, and formic acid (15/4/1) according to Dobrev and Kamínek (2002). An internal isotopic standard mixture consisting of deuterated IAA and ABA, and kinetin labelled with nitrogen ${ }^{15} \mathrm{~N}$ was added to each sample during homogenisation. The prepared extract was fractionated using solid phase extraction (SPE) columns Oasis MCX (Waters), and three fractions were collected: "acidic" eluted with methanol for IAA and ABA analyses according to HPLC method of Štefančič et al. (2007), "basic" eluted with methanolic solution of ammonia for cytokinins analyses according to Żur et al. (2015), and "flow through" for carbohydrates analyses according to Hura et al. (2016).

\section{Quantification of IAA and ABA}

After IAA and ABA elution from SPE column by pure methanol, each sample was evaporated to dryness and reconstituted in $50 \mu \mathrm{l}$ methanol (Żur et al. 2015). Finally, samples were analysed using a Supelco Ascentis RP-Amide column $(7.5 \mathrm{~cm} \times 2.1 \mathrm{~mm}, 2.7 \mu \mathrm{m})$. Mobile phases consisted of $0.1 \%$ formic acid solution in water (solvent $\mathrm{A}$ ) and acetonitrile:methanol (1:1) mixture (solvent B). Analyses were performed using gradient elution at a flow rate of $1.5 \mathrm{ml} \mathrm{min}{ }^{-1}$ with gradient starting from $20 \% \mathrm{~B}$ at $0 \mathrm{~min}$, rising to $80 \% \mathrm{~B}$ at $3 \mathrm{~min}$, and back to $20 \%$ at $3.5 \mathrm{~min}$, total analysis time was $4 \mathrm{~min}$. The HPLC apparatus was Agilent Technologies 1290 Infinity equipped with Agilent Technologies 6460 Triple Quad LC/MS with Jet Stream. Capillary voltage was set to $4000 \mathrm{~V}$, two the most abundant product ions were monitored using MRM (Multiple Reaction Monitoring) mode for each analysed compound. The most abundant product ion was used for quantification (quantifier), whereas the other ion was used to confirm the identity of the phytohormones (qualifier). The MRMs were set as follows in Supplementary Table 1. Agilent Technologies Mass Hunter 5 software was used for apparatus control, data collection, and processing.

\section{Quantification of cytokinins}

Cytokinins, such as t-Z, c-Z, t-ZR, c-ZR, Kin, KinR and iPA, were separated from the samples. Their sum was defined as the total content of cytokinins and indicated by the abbreviation CKs. For their structural similarity, cytokinins were categorised into Z-type (i.e. $\mathrm{t}-\mathrm{Z}+\mathrm{c}-\mathrm{Z}+\mathrm{t}-\mathrm{ZR}+\mathrm{c}-\mathrm{ZR})$.

Fractions of cytokinins were flushed out from the SPE column after collecting IAA and ABA. Impurities were first washed out with $0.35 \mathrm{M}$ ammonia in water and then cytokinins were eluted with $0.35 \mathrm{M}$ ammonia in $60 \%$ methanol (Żur et al. 2015). The collected fraction was evaporated to dryness, reconstituted in $50 \mu \mathrm{l}$ methanol, and analysed using the same chromatographic system and HPLC column as described above. The solvent system consisted of water with $0.001 \%$ acetic acid (solvent A) and acetonitrile with $0.001 \%$ acetic acid (solvent B) at a flow rate of $1.5 \mathrm{ml} \mathrm{min}{ }^{-1}$, gradient profile was $2.5 \% \mathrm{~B}$ to $1 \mathrm{~min}, 10 \%$ $\mathrm{B}$ at $3 \mathrm{~min}, 25 \% \mathrm{~B}$ at $6 \mathrm{~min}, 75 \% \mathrm{~B}$ at $8 \mathrm{~min}$ and $2.5 \% \mathrm{~B}$ at $8.5 \mathrm{~min}$. Capillary voltage was set to $4000 \mathrm{~V}$, two the most abundant positive secondary ions were monitored as follows in Supplementary Table 2.

\section{Quantification of soluble sugars}

The "flow through" fraction collected from the SPE column was lyophilised and reconstituted according to the 
procedure described by Janeczko et al. (2010). Samples were analysed on Agilent Technologies 1200 HPLC equipped with an amperometric detector ESA Coulochem II Analytical Cell 5040 with gold electrode. The Hamilton RCX-10 250×4.1 mm (Hamilton, Reno, NV, USA) HPLC column was used. The mobile phase comprised $100 \mathrm{mM}$ sodium hydroxide solution in water, and the flow rate was set to $1.5 \mathrm{ml} \mathrm{min}{ }^{-1}$. The detector was set at an analytical potential of $200 \mathrm{mV}$, an oxidising potential of $800 \mathrm{~m}$, and a reducing potential of $-900 \mathrm{mV}$ (with reference to a palladium electrode). Agilent Technologies ChemStation B04 software was used for apparatus control, data collection, and processing.

\section{Statistical analysis}

Data for endogenous hormone and sugar contents are presented as mean $\pm \mathrm{SD}$ for three replicates $(35 \mathrm{mg} \mathrm{FW}$, i.e. about 80 explants, was considered as one replicate). Statistical analysis for etiolated and non-etiolated explants was performed using the Student's $t$ test embedded in microsoft excel. Only a return of $\mathrm{p}<0.05$ was designated as being statistically significant. As the number of data for analysis conducted during the 14 days of culture was limited it was not possible to execute normality tests, thus a nonparametric Analysis of Variance (Kruskal Wallis test) was applied to calculate the significant differences between the comparisons (corrected $\mathrm{p} \leq 0.05$ ). The parameters that contributed significantly to the final score were determined by Multiple comparisons of mean ranks. All statistical analyses were performed using STATISTICA version 6.0 (Stat Soft Inc., USA, 2001) package.

\section{Results}

\section{The effect of etiolation on endogenous hormone and soluble sugar contents in initial explants}

The concentration of phytohormones and soluble sugars in stipe explants derived from sporophytes developed under photoperiodic conditions (non-etiolated) and in those developed in darkness (etiolated) was significantly different (Fig. 1). Etiolated explants (capable of SE) were characterised by almost 12-fold lower concentration of ABA, 2-fold greater IAA content and 9.4-fold greater concentration of total cytokinins in comparison with non-etiolated explants (incapable of SE; Fig. 1a, b). Moreover, the content of some individual cytokinins such as KinR and c-ZR was more than 450 times greater in etiolated explants than in non-etiolated ones. The concentrations of hexoses and sucrose were more than 2- and 39-fold lower in etiolated explants than in the plant material kept under photoperiodic conditions, respectively (Fig. 1b).
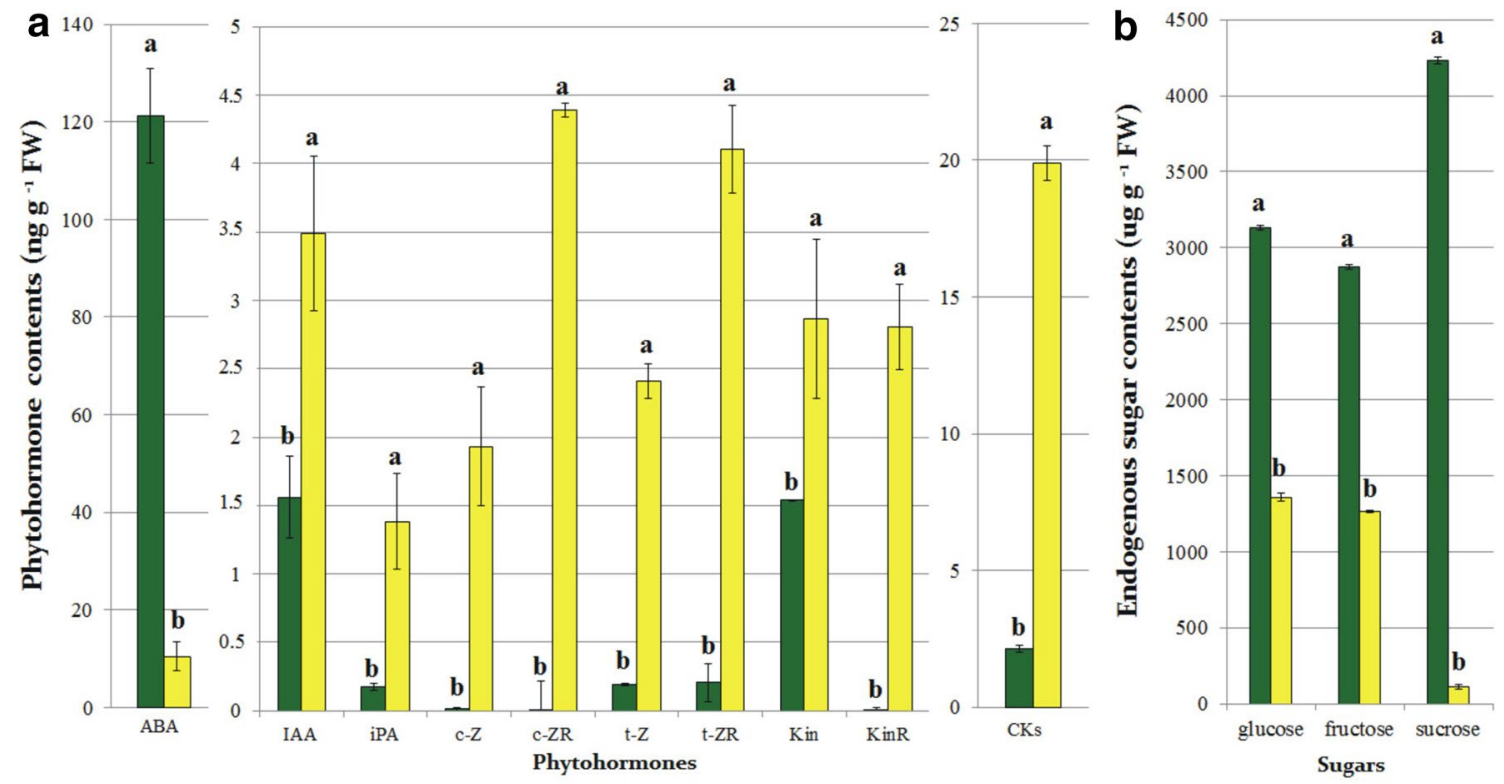

non-etiolated $\square$ etiolated

Fig. 1 Contents of $\mathbf{a}$ endogenous hormones and $\mathbf{b}$ soluble sugars in non-etiolated (incapable of somatic embryogenesis) and etiolated (capable of somatic embryogenesis) explants of $C$. delgadii. Data are the means $( \pm \mathrm{SD})$ of three replicates. The Student's $t$ test was used independently for each type of phytohormones and sugars. Mean values marked with the same letter do not differ significantly at the 0.05 confidence level. $A B A$ abscisic acid, $C K s$ the sum of c-Z, c-ZR, iPA, Kin, KinR, $\mathrm{t}-\mathrm{Z}$ and $\mathrm{t}-\mathrm{ZR}$ concentrations, $c-Z$ cis zeatin, $c-Z R$ cis zeatin riboside, IAA indole-3-acetic acid, $i P A$ isopentenyladenosine, Kin kinetin, $\operatorname{Kin} R$ kinetin riboside, $t-Z$ trans zeatin, $t-Z R$ trans zeatin riboside 
In order to assess the hormonal balance in both types of plant material, the interaction between endogenous hormones was expressed by their ratios (Table 1). In non-etiolated explants, the ratios of IAA and ABA to individual cytokinins (also to CKs), as well as ABA to IAA, were much greater than those in etiolated ones. This was especially evident in the case of KinR, c-ZR and c-Z. Etiolation of donor plantlets resulted in equalisation of all ratios mentioned above (excluding IAA/Kin). Only in case of the ratio of Z-type cytokinin to iPA, its value was lower in non-etiolated explants (2.4) than in etiolated explants (9.3) (Table 1).

\section{Changes in endogenous hormone and soluble sugar contents during 14-day-long culture}

To characterise the physiological changes that occur during early SE in etiolated explant of $C$. delgadii, the content of endogenous hormones (Fig. 2a-e) and sugars (Fig. 2f) was

Table 1 Ratios of different phytohormones in non-etiolated and etiolated initial explants of $C$. delgadii

\begin{tabular}{lccc}
\hline $\begin{array}{l}\text { Phytohormone } \\
\text { ratios }\end{array}$ & $\begin{array}{l}\text { Non-etiolated } \\
\text { explants }\end{array}$ & $\begin{array}{l}\text { Etiolated } \\
\text { explants }\end{array}$ & $\begin{array}{l}\text { Non-etiolated/ } \\
\text { etiolated explants }\end{array}$ \\
\hline IAA/CKs & $\mathbf{0 . 7 3} \pm \mathbf{0 . 1 a}$ & $\mathbf{0 . 1 8} \pm \mathbf{0 . 1 b}$ & $\mathbf{4 . 0}$ \\
IAA/t-Z & $8.1 \pm 2.1 \mathrm{a}$ & $1.5 \pm 0.7 \mathrm{~b}$ & 5.4 \\
IAA/c-Z & $145.1 \pm 13.9 \mathrm{a}$ & $1.8 \pm 1.1 \mathrm{~b}$ & 80.6 \\
IAA/t-ZR & $7.6 \pm 2.8 \mathrm{a}$ & $0.9 \pm 0.5 \mathrm{~b}$ & 8.4 \\
IAA/c-ZR & $159.6 \pm 6.9 \mathrm{a}$ & $0.8 \pm 0.5 \mathrm{~b}$ & 199.5 \\
IAA/Kin & $1.0 \pm 0.1 \mathrm{a}$ & $1.2 \pm 0.2 \mathrm{~b}$ & 0.8 \\
IAA/KinR & $435.3 \pm 12.4 \mathrm{a}$ & $1.2 \pm 0.5 \mathrm{~b}$ & 362.8 \\
IAA/iPA & $9.0 \pm 1.9 \mathrm{a}$ & $2.5 \pm 0.5 \mathrm{~b}$ & 3.6 \\
ABA/CKs & $\mathbf{5 6 . 8} \pm \mathbf{3 . 2 a}$ & $\mathbf{0 . 5 3} \pm \mathbf{0 . 2 b}$ & $\mathbf{1 0 7 . 2}$ \\
ABA/t-Z & $626.3 \pm 44.1 \mathrm{a}$ & $4.3 \pm 1.9 \mathrm{~b}$ & 145.7 \\
ABA/c-Z & $11,275.9 \pm 63.1 \mathrm{a}$ & $5.4 \pm 1.6 \mathrm{~b}$ & 2088.1 \\
ABA/t-ZR & $591.8 \pm 16.6 \mathrm{a}$ & $2.6 \pm 0.7 \mathrm{~b}$ & 227.6 \\
ABA/c-ZR & $12,400.5 \pm 24.1 \mathrm{a}$ & $2.4 \pm 0.6 \mathrm{~b}$ & 5166.9 \\
ABA/Kin & $78.9 \pm 9.7 \mathrm{a}$ & $3.7 \pm 2.0 \mathrm{~b}$ & 21.3 \\
ABA/KinR & $33,829.6 \pm 80.6 \mathrm{a}$ & $3.7 \pm 1.8 \mathrm{~b}$ & 9143.1 \\
ABA/iPA & $\mathbf{6 9 5 . 8 \pm 2 4 . 2 \mathrm { a }}$ & $7.6 \pm 3.0 \mathrm{~b}$ & 91.6 \\
ABA/IAA & $\mathbf{7 7 . 7} \pm \mathbf{1 2 . 0 a}$ & $\mathbf{2 . 7} \pm \mathbf{0 . 4 b}$ & $\mathbf{2 8 . 8}$ \\
Z-type/iP-type & $\mathbf{2 . 4} \pm \mathbf{0 . 4 a}$ & $\mathbf{9 . 3} \pm \mathbf{4 . 9 b}$ & $\mathbf{0 . 3}$ \\
\hline
\end{tabular}

Data are the means $( \pm \mathrm{SD})$ of three replicates. The Student's $t$ test was used independently for each type of ratio determined. Mean values marked with the same letter do not differ significantly at the 0.05 confidence level

Values marked with the same letter differ insignificantly at the 0.05 confidence level according to Student's $t$ test

$A B A$ abscisic acid, $C K s$ the sum of c-Z, c-ZR, Kin, KinR, t-Z, t-ZR, and iPA concentrations, $c-Z$ cis zeatin, $c-Z R$ cis zeatin riboside, $I A A$ indole-3-acetic acid, $i P A$ isopentenyladenosine, Kin kinetin, KinR kinetin riboside, $t-Z$ trans zeatin, $t-Z R$ trans zeatin riboside, $Z$-type the sum of c-Z, c-ZR, t-Z, t-ZR concentrations determined during 14 days of culture. The content of all phytohormones studied diminished rapidly after excision of explant (i.e. at day 2 of culture). Between days 4 and 14, their concentrations remained relatively stable, excluding IAA, whose concentration decreased at day 10 (Fig. 2b), and Kin, whose concentration increased at day 12 (Fig. 2e), and t-ZR, whose amount increased starting on day 8 of culture (Fig. 2d).

The hormonal balance during induction of SE is presented in Table 2. Results showed that the ratios of IAA to the individual cytokinins (as well as to CKs) increased reaching its maximum at day 6 of culture. The ratio of Z-type cytokinin to iPA was significantly lower between days 2 and 8 of culture. After that, its value increased gradually. The values of the ABA/CKs (as well as to individual cytokinins) and $\mathrm{ABA} / \mathrm{IAA}$ ratios remained almost unchanged during 14-day-long culture of stipe explants (Table 2).

The explant excision did not influence significantly the content of endogenous sugars (Fig. 2f). Intensive sucrose accumulation (with a mean 12-fold increase) was observed at day 6 of culture. Over the next 2 days, the level diminished by $37 \%$, and remained relatively stable up to day 14 . An increase in the hexose concentrations was observed at day 4 . Then, content of both the glucose and fructose gradually diminished.

\section{Discussion}

The morphogenetic response of plant material in vitro is greatly influenced by the ontogenetic age of the tissue or organ which is used as the source of the initial explant (Gaj 2004). Along with changes in the degree of tissue differentiation, the physiological state of cells is modified. The basis of these changes is an altered pattern of endogenous hormone synthesis, conjugation and/or inactivation, which can be modulated by the use of many stress-related factors (Fehér 2015) as well as by etiolation and explant excision.

\section{Etiolation resulting in altered concentration and balance of endogenous hormones}

Light affects the biosynthesis and signalling of endogenous hormones (Symons and Reid 2003). In cryptogamic plants, light is a crucial factor in controlling the regeneration pathways, including SE (Mikuła et al. 2015a). Therefore, we carried out quantitative analysis of ABA, IAA and cytokinin contents in etiolated (capable of SE) and nonetiolated (incapable of SE) stipe explants of $C$. delgadii, and found extreme differences in both the hormone content and hormonal balance. Our studies showed significantly lower concentration of $\mathrm{ABA}$ in etiolated plant material. 


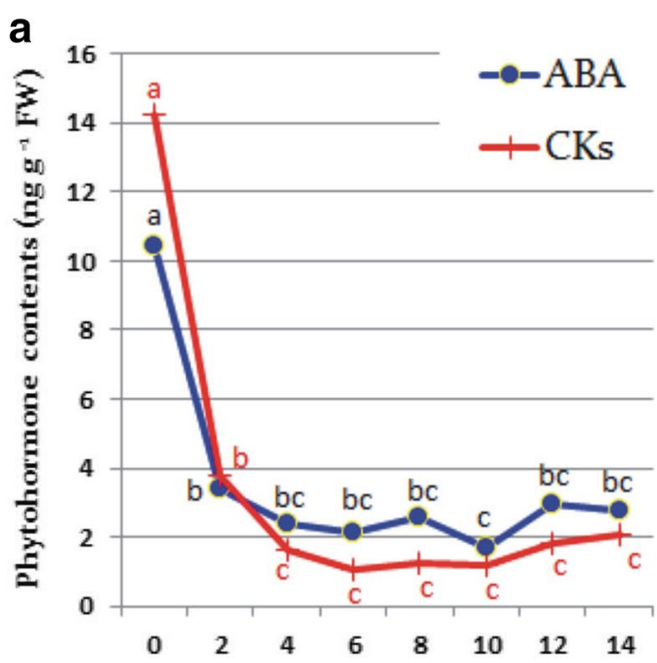

d
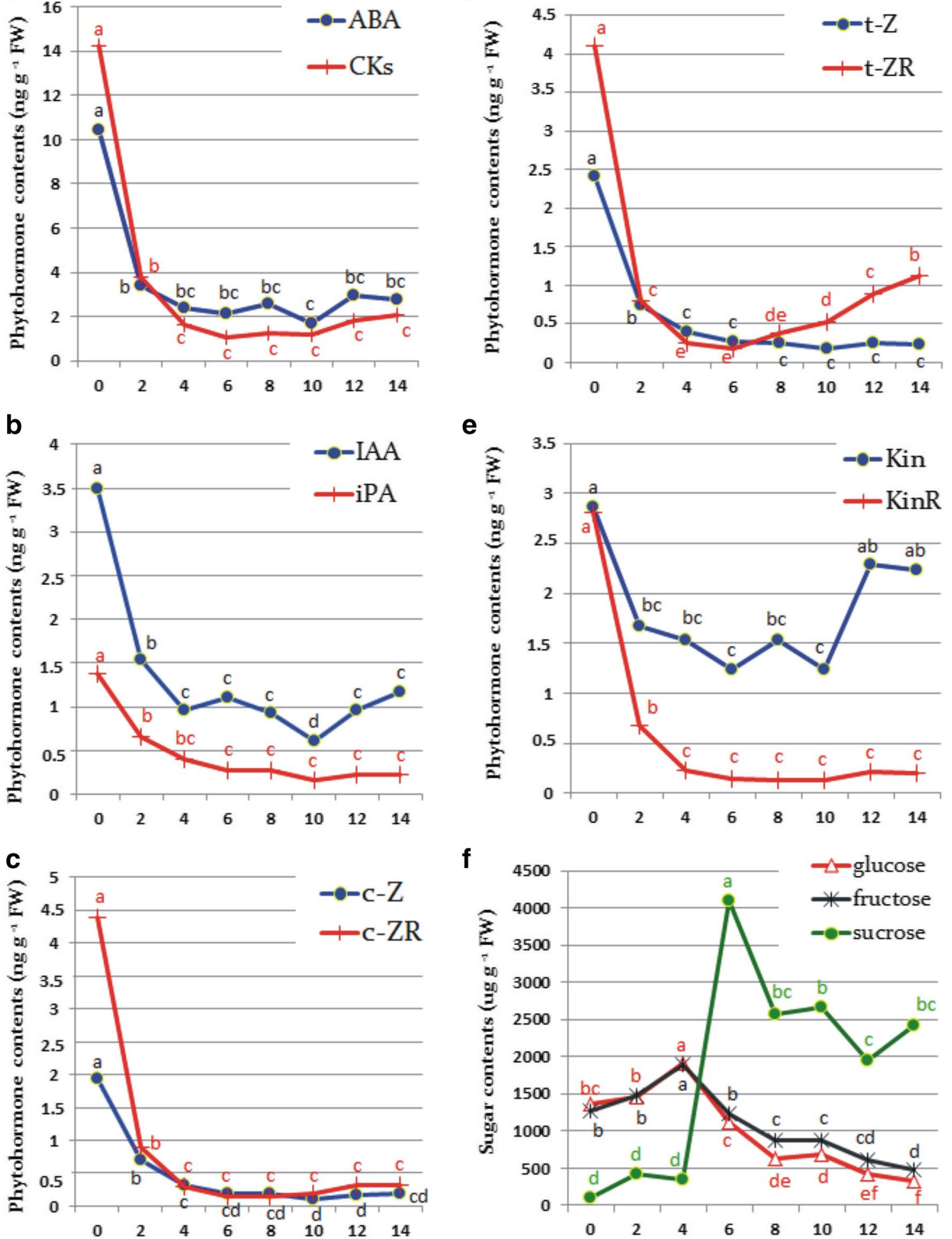

e

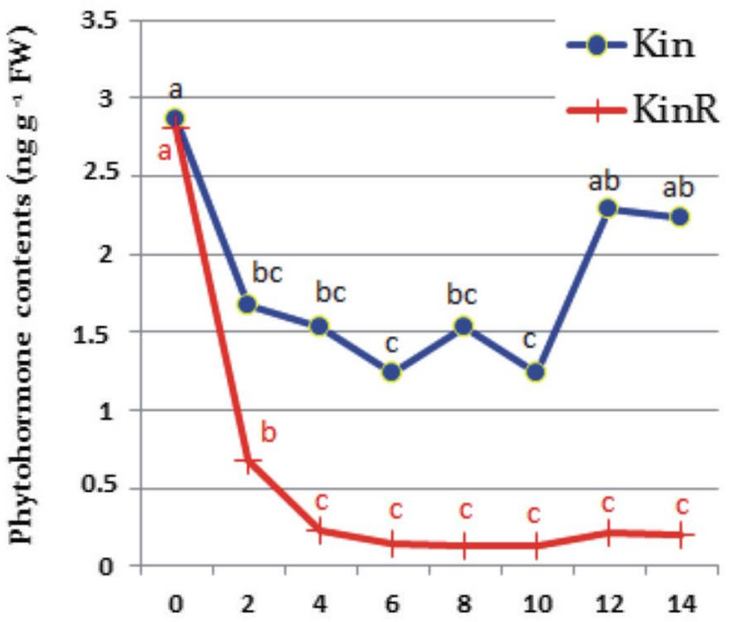

f

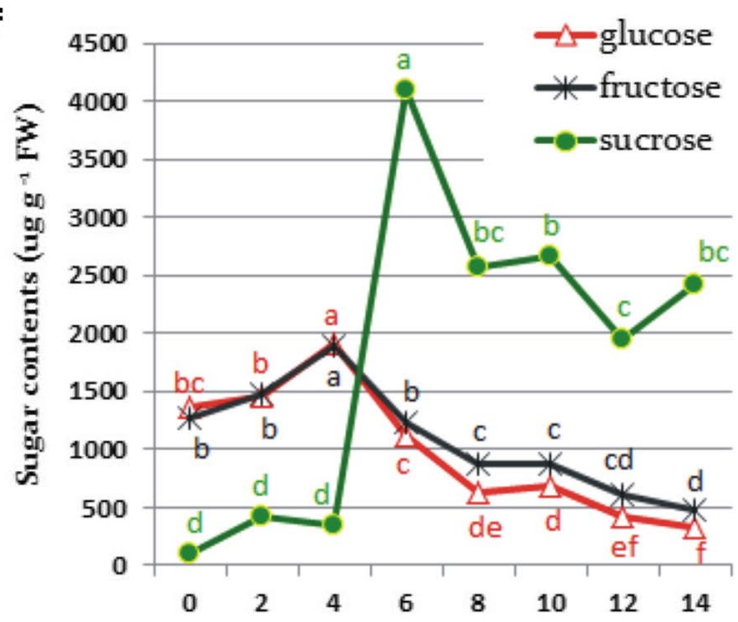

\section{Day of culture}

Fig. 2 Concentrations of phytohormones and soluble sugars during early somatic embryogenesis (14 days of culture in darkness). Data are the means of three replicates. Mean values marked with the same letter do not differ significantly at the 0.05 confidence level according to Kruskal-Wallis ANOVA and Median test. $A B A$ abscisic acid, $C K S$ the sum of c-Z, c-ZR, iPA, Kin, KinR, t-Z and t-ZR concentrations, $c-Z$ cis zeatin, $c-Z R$ cis zeatin riboside, $I A A$ indole-3-acetic acid, $i P A$ isopentenyladenosine, $\operatorname{Kin}$ kinetin, $K i n R$ kinetin riboside, $t-Z$ trans zeatin, $t-Z R$ trans zeatin riboside 
Table 2 Ratios of different phytohormones in etiolated explants during 14-day-long culture of C. delgadii

\begin{tabular}{|c|c|c|c|c|c|c|c|c|}
\hline \multirow{2}{*}{$\begin{array}{l}\text { Phytohormone } \\
\text { ratios }\end{array}$} & \multicolumn{8}{|c|}{ Day of culture } \\
\hline & 0 & 2 & 4 & 6 & 8 & 10 & 12 & 14 \\
\hline IAA/CKs & $0.5 \pm 0.06 c$ & $0.7 \pm 0.01 b$ & $0.8 \pm 0.02 b$ & $1.4 \pm 0.16 a$ & $1.0 \pm 0.16 b$ & $0.8 \pm 0.12 b$ & $0.6 \pm 0.04 c$ & $0.8 \pm 0.01 c$ \\
\hline IAA/t-Z & $1.4 \pm 0.50 \mathrm{c}$ & $2.0 \pm 0.09 \mathrm{c}$ & $2.4 \pm 0.22 b c$ & $4.2 \pm 1.73 \mathrm{ab}$ & $3.9 \pm 0.43 b$ & $3.5 \pm 1.31 b$ & $3.6 \pm 0.44 b$ & $4.9 \pm 0.74 a$ \\
\hline IAA/c-Z & $1.8 \pm 0.79 \mathrm{~d}$ & $2.1 \pm 0.13 \mathrm{~d}$ & $3.1 \pm 0.37 \mathrm{c}$ & $6.17 \pm 2.5 \mathrm{a}$ & $5.5 \pm 2.0 \mathrm{~b}$ & $5.7 \pm 1.5 \mathrm{ab}$ & $5.6 \pm 1.5 \mathrm{ab}$ & $6.1 \pm 0.5 \mathrm{a}$ \\
\hline IAA/t-ZR & $0.8 \pm 0.37 \mathrm{c}$ & $2.0 \pm 0.17 b c$ & $3.8 \pm 0.75 b$ & $5.9 \pm 1.74 \mathrm{a}$ & $2.4 \pm 0.28 b$ & $1.2 \pm 0.21 \mathrm{c}$ & $1.1 \pm 0.48 \mathrm{c}$ & $1.0 \pm 0.1 \mathrm{c}$ \\
\hline IAA/c-ZR & $0.8 \pm 0.37 \mathrm{e}$ & $1.7 \pm 0.1 \mathrm{~d}$ & $3.1 \pm 0.38 \mathrm{c}$ & $7.3 \pm 2.22 \mathrm{a}$ & $6.7 \pm 1.13 b$ & $3.6 \pm 1.81 \mathrm{c}$ & $3.0 \pm 0.4 \mathrm{c}$ & $3.8 \pm 0.49 \mathrm{c}$ \\
\hline IAA/iPA & $2.4 \pm 0.39 \mathrm{c}$ & $2.3 \pm 0.17 \mathrm{c}$ & $2.4 \pm 0.05 \mathrm{c}$ & $4.2 \pm 1.66 \mathrm{ab}$ & $3.5 \pm 0.54 b$ & $3.9 \pm 1.11 b$ & $4.2 \pm 0.51 \mathrm{ab}$ & $5.4 \pm 0.69 a$ \\
\hline IAA/Kin & $1.2 \pm 0.17 \mathrm{a}$ & $0.9 \pm 0.14 \mathrm{ab}$ & $0.6 \pm 0.02 b$ & $0.9 \pm 0.30 \mathrm{ab}$ & $0.6 \pm 0.15 \mathrm{ab}$ & $0.6 \pm 0.39 \mathrm{ab}$ & $0.4 \pm 0.03 \mathrm{ab}$ & $0.5 \pm 0.02 \mathrm{ab}$ \\
\hline IAA/KinR & $1.2 \pm 0.38 \mathrm{~d}$ & $2.3 \pm 0.19 \mathrm{c}$ & $4.1 \pm 0.60 \mathrm{~b}$ & $8.3 \pm 3.91 \mathrm{a}$ & $7.8 \pm 2.40 \mathrm{ab}$ & $5.5 \pm 3.35 b$ & $4.5 \pm 0.09 b$ & $6.1 \pm 1.17 \mathrm{ab}$ \\
\hline $\mathbf{A B A} / \mathbf{C K s}$ & $0.5 \pm 0.17 b$ & $0.6 \pm 0.23 a b$ & $0.7 \pm 0.22 \mathrm{ab}$ & $0.9 \pm 0.32 a$ & $0.9 \pm 0.39 a$ & $0.7 \pm 0.13 a b$ & $0.7 \pm$ 0.38ab & $0.6 \pm 0.17 a b$ \\
\hline $\mathrm{ABA} / \mathrm{t}-\mathrm{Z}$ & $4.4 \pm 1.32 \mathrm{c}$ & $4.7 \pm 0.96 c$ & $6.0 \pm 0.86 b c$ & $7.7 \pm 0.97 b$ & $10.7 \pm 2.3 \mathrm{a}$ & $9.4 \pm 1.67 \mathrm{ab}$ & $11.0 \pm 0.71 \mathrm{a}$ & $11.3 \pm 0.15 \mathrm{a}$ \\
\hline $\mathrm{ABA} / \mathrm{c}-\mathrm{Z}$ & $5.4 \pm 1.12 \mathrm{e}$ & $5.0 \pm 0.93 \mathrm{e}$ & $7.7 \pm 1.3 \mathrm{~d}$ & $11.2 \pm 1.39 \mathrm{c}$ & $15.9 \pm 7.3 \mathrm{bc}$ & $15.4 \pm 1.14 b$ & $17.1 \pm 3.66 \mathrm{a}$ & $14.1 \pm 0.77 b$ \\
\hline $\mathrm{ABA} / \mathrm{t}-\mathrm{ZR}$ & $2.6 \pm 0.52 \mathrm{c}$ & $4.7 \pm 1.6 b c$ & $9.5 \pm 2.35 \mathrm{a}$ & $11.1 \pm 2.59 \mathrm{a}$ & $6.7 \pm 0.14 b$ & $3.2 \pm 0.04 \mathrm{c}$ & $3.4 \pm 1.32 \mathrm{c}$ & $2.5 \pm 0.58 \mathrm{c}$ \\
\hline $\mathrm{ABA} / \mathrm{c}-\mathrm{ZR}$ & $2.4 \pm 0.42 \mathrm{e}$ & $4.1 \pm 1.27 \mathrm{~d}$ & $7.8 \pm 1.36 \mathrm{c}$ & $13.8 \pm 3.13 b$ & $19.01 \pm 5.1 \mathrm{a}$ & $9.53 \pm 2.961 \mathrm{c}$ & $9.4 \pm 0.68 \mathrm{c}$ & $8.8 \pm 0.08 \mathrm{c}$ \\
\hline $\mathrm{ABA} / \mathrm{iPA}$ & $8.4 \pm 4.3 \mathrm{c}$ & $5.4 \pm 0.93 \mathrm{~d}$ & $5.9 \pm 0.43 \mathrm{~d}$ & $7.7 \pm 1.04 \mathrm{c}$ & $9.8 \pm 2.49 \mathrm{~b}$ & $10.6 \pm 0.9 \mathrm{ab}$ & $12.9 \pm 0.81 \mathrm{a}$ & $12.5 \pm 0.13 \mathrm{a}$ \\
\hline $\mathrm{ABA} / \mathrm{Kin}$ & $4.1 \pm 2.17 \mathrm{a}$ & $2.1 \pm 0.18 b$ & $1.5 \pm 0.12 b$ & $1.7 \pm 0.34 \mathrm{~b}$ & $1.8 \pm 0.60 \mathrm{~b}$ & $1.6 \pm 0.66 \mathrm{~b}$ & $1.3 \pm 0.03 b$ & $1.2 \pm 0.11 b$ \\
\hline ABA/KinR & $3.9 \pm 1.31 \mathrm{e}$ & $5.5 \pm 1.84 \mathrm{~d}$ & $10.2 \pm 2.01 \mathrm{c}$ & $14.7 \pm 0.84 b$ & $22.3 \pm 9.07 \mathrm{a}$ & $14.2 \pm 6.03 b$ & $14.2 \pm 0.58 b$ & $14.0 \pm 0.77 b$ \\
\hline ABA/IAA & $2.7 \pm 0.4 a$ & $2.2 \pm 0.8 \mathrm{ab}$ & $2.5 \pm 0.2 \mathrm{a}$ & $1.9 \pm 0.5 \mathrm{ab}$ & $2.8 \pm 0.4 a$ & $2.8 \pm 0.8 a$ & $3.0 \pm 0.3 a$ & $2.3 \pm 0.5 a b$ \\
\hline Z-type/iPA & $9.9 \pm 3.1 \mathrm{a}$ & $4.8 \pm 0.4 \mathrm{c}$ & $3.2 \pm 0.3 \mathrm{c}$ & $2.9 \pm 0.1 \mathrm{c}$ & $3.6 \pm 0.3 c$ & $6.3 \pm 0.0 b$ & $7.5 \pm 1.6 a$ & $8.6 \pm 1.2 \mathrm{a}$ \\
\hline
\end{tabular}

Data are the means $( \pm \mathrm{SD})$ of three replicates. Mean values marked with the same letter do not differ significantly at the 0.05 confidence level according to Kruskal-Wallis ANOVA and Median test

Values marked with the same letter differ insignificantly at the 0.05 confidence level according to Kruskal-Wallis ANOVA and Median test

$A B A$ abscisic acid, $C K s$ the sum of c-Z, c-ZR, Kin, KinR, t-Z, t-ZR, and iPA concentrations, $c-Z$ cis zeatin, $c$-ZR cis zeatin riboside, IAA indole3 -acetic acid, $i P A$ isopentenyladenosine, Kin kinetin, KinR kinetin riboside, $t$ - $Z$ trans zeatin, $t$ - $Z R$ trans zeatin riboside, $Z$-type the sum of c-Z, $\mathrm{c}-\mathrm{ZR}, \mathrm{t}-\mathrm{Z}, \mathrm{t}-\mathrm{ZR}$ concentrations

The results are in agreement with previously published data (Volmaro et al. 1998; Pacholczak et al. 2005). ABA is biosynthesised especially in chloroplasts and appears to be a cleavage product in the biosynthetic pathway of carotenoids (Seo and Koshiba 2002). In view of this, the reduction in ABA concentration in response to darkness seems to be related to changes in the structure and/or function of chloroplasts. Researchers also agree that the exposure of plants to darkness increases the concentration of endogenous IAA, even twice (Liu et al. 1996; Volmaro et al. 1998; Suzuki and Kerbauy 2006), and it is in agreement with the results obtained for $C$. delgadii. The negative effect of the light on IAA concentration is presumably because light induces IAA-peroxidase and IAA-oxidase activity, the key enzymes involved in the breaking down of IAA (Liu et al. 1996). Dark treatment also greatly stimulates cytokinin accumulation. In shoots of dark-grown Catasetum fimbriatum, the total cytokinin concentration was almost threefold greater than in those growing under photoperiodic conditions (Suzuki and Kerbauy 2006). In stipe explants of $C$. delgadii, this difference reached more than 9 in favour of the dark. Similarly, the concentrations of Z, ZR, iP, and iPA were significantly higher after the exposure to darkness than to continuous light (Macháčková et al. 1996). Our data confirmed this to be true also for other cytokinins, i.e. Kin and its riboside. Although the occurrence of Kin has been considered to be an artefact, its presence had already been found in plant material such as coconut chunks (Barciszewski et al. 1996) and water extracts (Ge et al. 2005), root nodules of Australian pine (Raman and Elumalai 1996) as well as triticale anthers (Żur et al. 2015). The importance of Kin in controlling different physiological processes in plants may be associated with its antioxidant and ABAantagonistic properties (Barciszewski et al. 1996; Żur et al. 2015).

The key question of our research is how the content/ratio of phytohormones, established as a result of etiolation of the donor plantlets, promotes SE induction? The hormonal state of somatic cells determines the morphogenetic response of explants by genes classified as hormone-related transcription factors (Gliwicka et al. 2013). Some authors postulate that elevated level of ABA reduces/inhibits the explant ability to SE (Ivanova et al. 1994; Jiménez and Bangerth 2001a). According to this, our results showed the many times higher $\mathrm{ABA} / \mathrm{CKs}$ and $\mathrm{ABA} / \mathrm{IAA}$ ratios in non-embryogenic explants than in embryogenic explants. On the level of molecular studies, the negative relationship between the ABA content and the LEC2 (LEAFY 
COTYLEDON2) activity during SE induction was recently revealed by Wójcikowska and Gaj (2015). On the other hand, LEC2 activation induces YUC2 and YUC4 (YUCCA 2 and 4) genes involved in auxin biosynthesis (Stone et al. 2008). The connection between auxin and genes that are specific for early SE, such as AGL15 (AGAMOUS-Like15) and FUS3 (FUSCA3) has also been documented (Stone et al. 2008; Zheng et al. 2013). It is commonly known that an appropriate level of auxin is required for SE induction (Ayil-Gutiérrez et al. 2013; Gliwicka et al. 2013). Thus, the competence of $C$. delgadii explants to undergo SE may relate to low ABA level together with high content of IAA and cytokinins achieved by etiolation.

\section{Explant excision resulting in altered endogenous hormone concentration}

Our earlier research revealed that the tissue damage caused by aging and dying of the upper part of etiolated fronds can spontaneously induce direct or indirect SE in $C$. delgadii on PGR-free medium (Mikuła et al. 2015b). In plant tissue culture, wounding is one of the most important factors affecting the developmental programme of somatic cells (Iwase et al. 2011). Sometimes, this stress treatment may be enough to initiate SE (Smith and Krikorian 1989). Following a wound, the level of IAA in tobacco leaves declined 2to threefold (Thornburg and Li 1991). Moreover, enhanced endogenous ABA, jasmonic acid, and ethylene production were detected (León et al. 2001). Wounding also enhanced the endogenous cytokinin activity (Crane and Ross 1986; Iwase et al. 2011). However, the hormonal response to excision seems to be completely different than to wounding. An excision releases explants from the influence of apical meristems that are the sources of hormone synthesis. Therefore, in excised stipes of $C$. delgadii about threefold reduction in the concentration of IAA, ABA and CKs was observed. The similar decline in IAA content was also noticed in excised cotton hypocotyl explants (Yang et al. 2012). Furthermore, it is worth mentioning that although a dramatic decline in the hormone concentration occurred, the balance between IAA/CKs, $\mathrm{ABA} / \mathrm{CKs}$ and $\mathrm{ABA} / \mathrm{IAA}$ ratios remained almost unchanged, and significantly greater concentration of CKs than IAA was still maintained in $C$. delgadii explants.

\section{Summary of physiological events during early SE of $C$. delgadii}

Analysis of the induction phase of SE from the viewpoint of physiological changes reveals three sub-phases (I-III) that can be observed in $C$. delgadii explants, and these are triggered by etiolation, explant excision, and a massive increase in endogenous sucrose concentration (Fig. 3).
Our results provide evidence that by etiolation of donor plantlets it is possible to achieve the hormonal balance which requires only a slight further modification to switch the developmental path of somatic cells. The etiolation reduces a high concentration of $\mathrm{ABA}$, the main factor inhibiting somatic embryo production (Charrière et al. 1999; Jiménez and Bangerth 2001a), and increases the content of IAA and cytokinins. As a result of explant excision a dramatic decline in the concentration of studied hormones occurs, but the hormonal balance and sugar content remain almost unchanged. Among those phytohormones, the rapid decline in IAA concentration seems to be directly related to the sugar influx into the explant cells. It was shown that the low level of IAA leads to an increase in apoplastic $\mathrm{pH}$ and the activation of invertases (Mohr and Schopfer 1995). Seeing that, almost 12 -fold increase in sucrose concentration due to the influx of glucose and fructose into the explant cells of $C$. delgadii, may represent an osmotic signal that is required for the expression of embryogenic competence (Kikuchi et al. 2006). We suggest that the short-term osmotic signal is a final switch to the massive genetic reprogramming towards embryogenesis. The studies also showed that the predominant class of hormones found in the stipe explants during early SE are cytokinins. In all likelihood, they favour this type of morphogenetic response, however, initiation of third sub-phase of induction phase seems to be supervised by auxin, as apparent from the highest IAA/CKs ratio on day 6 of culture.

In $C$. delgadii, the hormonal balance established at 6th day of culture (Fig. 3) promotes transition from the induction to the expression phase four days later. During next four days of culture many somatic embryos at the linear stage of development were present. This phase requires a considerable amount of metabolic energy and carbon skeletons (Gomes et al. 2014; Cangahuala-Inocente et al. 2009). Our results confirmed it by observation of intensive utilisation of soluble sugars. Third part of acquisition phase and an early stage of SE expression were also correlated with a greater concentration of cytokinins Z-type compared with iPA. This result resembles previous data indicating that the ratio of these two types of cytokinins is a good index of both the explant and callus embryogenic capacity (Centeno et al. 1997; Pintos et al. 2002). Among Z-type cytokinins, t-ZR appears to be particularly associated with frequent cell divisions leading to the production of linear stage somatic embryos in C. delgadii.

\section{Conclusion}

1. The capacity of stipe explants to form somatic embryos in $C$. delgadii is determined by the endogenous level and balance of hormones that are modulated by the 


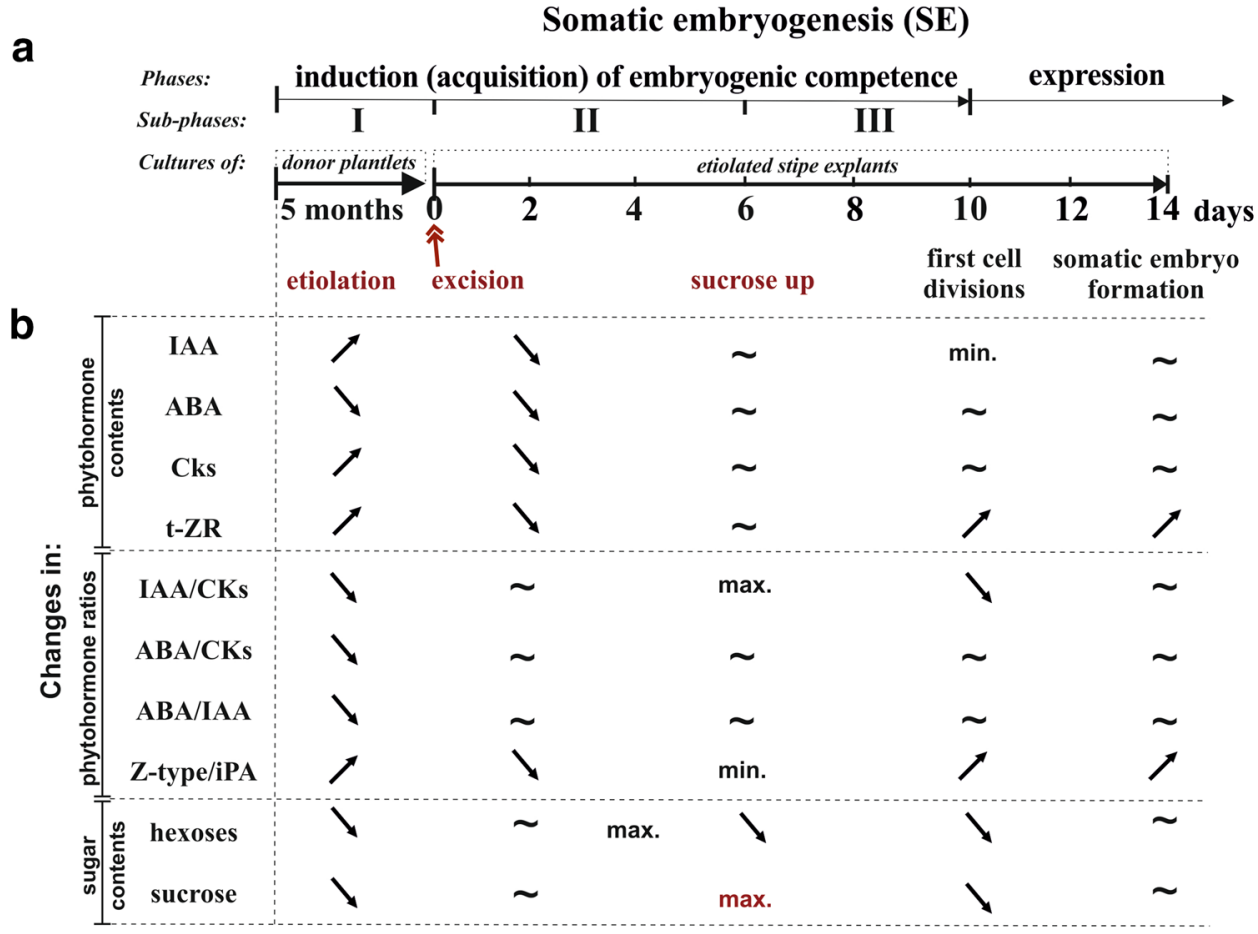

Fig. 3 Schematic summary of physiological events happened during early somatic embryogenesis in $C$. delgadii. a Time-line of embryogenic pathway development illustrating the two different phases, i.e. the induction (acquisition) of embryogenic competence and the expression of SE. The acquisition phase is divided into three subphases (I-III). There are also three different stimuli: etiolation, excision (exogenously applied), and a massive increase in endogenous sucrose concentration (sucrose $u p$ ). They switch these sub-phases on

conditions of light under which the donor plantlets have developed.

2. Drastic reduction in hormone contents caused by excision is sufficient to trigger embryogenic competence in epidermal cells of $C$. delgadii stipe explants.

3. Rapid increase in sucrose content can be considered as a switch to the SE expression phase.

4. Trans-zeatin riboside is clearly associated with SE expression phase of $C$. delgadii in which the frequent cell divisions happen and the somatic embryos at linear stage appear.

Acknowledgements The authors thank Professor Jan J. Rybczyński and Dr. Karolina Tomiczak (PAS Botanical Garden CBDC in Powsin, Warsaw, Poland) for their valuable advice and creative discussions during preparation of the manuscript. We would like to give special thanks to anonymous Reviewer 2 for valuable comments and suggestions. This research was supported by the Polish National Science Centre (NCN), no. 2011/03/B/NZ9/02472.

Author Contributions MG performed the experiments and prepared the samples for HPLC analysis. AK performed HPLC analyses of carbohydrates, PW carried out HPLC separation of phytohormones. AK and PW performed all chromatographic data analysis. and off. b Changes in endogenous hormone and sugar contents, and in hormone ratios, showing the influence of the stimuli. $A B A$ abscisic acid, $C K s$ the sum of c-Z, c-ZR, iPA, Kin, KinR, t-Z and t-ZR concentrations, $c-Z$ cis zeatin, $c-Z R$ cis zeatin riboside, $I A A$ indole-3-acetic acid, $i P A$ isopentenyladenosine, $\operatorname{Kin}$ kinetin, $\operatorname{Kin} R$ kinetin riboside, $t-Z$ trans zeatin, $t$ - $Z R$ trans zeatin riboside, $Z$-type the sum of $\mathrm{t}-\mathrm{Z}, \mathrm{c}-\mathrm{Z}$, $\mathrm{t}-\mathrm{ZR}$, c-ZR concentrations, min./max. minimum/maximum content, $\uparrow / \downarrow / \sim$ increasing/decreasing/constant trend

MG and AM designed the experiments and wrote the manuscript. AM conceived the study. All authors read and approved the final manuscript.

\section{Compliance with ethical standards}

Conflict of interest The authors declare that they have no conflict of interest.

Open Access This article is distributed under the terms of the Creative Commons Attribution 4.0 International License (http:// creativecommons.org/licenses/by/4.0/), which permits unrestricted use, distribution, and reproduction in any medium, provided you give appropriate credit to the original author(s) and the source, provide a link to the Creative Commons license, and indicate if changes were made.

\section{References}

Ayil-Gutiérrez B, Galaz-Avalos RM, Peña-Cabrera E, Loyola-Vargas VM (2013) Dynamics of the concentration of IAA and some of its conjugates during the induction of somatic embryogenesis in Coffea canephora. Plant Signal Behav 8:1-10 
Barciszewski J, Siboska GE, Pedersen BO, Clark BF, Rattan SI (1996) Evidence for the presence of kinetin in DNA and cell extracts. FEBS Lett 393:197-200

Cangahuala-Inocente GC, Steiner N, Maldonado SB, Guerra MP (2009) Patterns of protein and carbohydrate accumulation during somatic embryogenesis of Acca sellowiana. Pesq Agropec Bras 44:217-224

Centeno ML, Rodríguez R, Berros B, Rodríguez A (1997) Endogenous hormonal content and somatic embryogenic capacity of Corylus aveliana L. cotyledons. Plant Cell Rep 17:139-144. doi:10.1007/s002990050367

Charrière F, Sotta B, Miginiac É, Hahne G (1999) Induction of adventitious shoots or somatic embryos on in vitro cultured zygotic embryos of Helianthus annuus: Variation of endogenous hormone levels. Plant Physiol Biochem 37:751-757. doi:10.1016/ S0981-9428(00)86688-7

Chen X, Cheng J, Chen L, Zhang G, Huang H, Zhang Y, Xu L (2016) Auxin-independent NAC pathway acts in response to explant-specific wounding and promotes root tip emergence during de novo root organogenesis in Arabidopsis. Plant Physiol. doi:10.1104/pp.15.01733

Crane KE, Ross CW (1986) Effects of wounding on cytokinin activity in cucumber cotyledons. Plant Physiol 82:1151-1152. doi:10.1104/pp.82.4.1151

Dobrev PI, Kamínek M (2002) Fast and efficient separation of cytokinins from auxin and abscisic acid and their purification using mixed-mode solid-phase extraction. J Chromatogr A 950:21-29. doi:10.1016/S0021-9673(02)00024-9

Elhiti M, Stasolla C, Wang A (2013) Molecular regulation of plant somatic embryogenesis. Vitr Cell Dev Biol - Plant 49:631-642. doi:10.1007/s11627-013-9547-3

Eveland AL, Jackson DP (2011) Sugars, signalling, and plant development. J Exp Bot 63:3367-3377. doi:10.1093/jxb/err379

Fehér A (2015) Somatic embryogenesis-stress-induced remodeling of plant cell fate. Biochim Biophys Acta 1849:385-402. doi:10.1016/j.bbagrm.2014.07.005

Fiore MC, Trabace T, Sunseri F (1997) High frequency of plant regeneration in sunflower from cotyledons via somatic embryogenesis. Plant Cell Rep 16:295-298. doi:10.1007/s002990050226

Gaj MD (2004) Factors influencing somatic embryogenesis induction and plant regeneration with particular reference to Arabidopsis thaliana (L.) Heynh. Plant Growth Regul 43:27-47. doi:10.1023/ B:GROW.0000038275.29262.fb

Ge L, Yong JWH, Goh NK, Chia LS, Tan SN, Ong LS (2005) Identification of kinetin and kinetin riboside in coconut (Cocos nucifera L.) water using a combined approach of liquid chromatography-tandem mass spectrometry, high performance liquid chromatography and capillary electrophoresis. J Chromatogr B 829:26-34. 10.1016/j.jchromb.2005.09.026

Gibson SI (2004) Sugar and phytohormone response pathways: navigating a signalling network. J Exp Bot 55:253-264. doi:10.1093/ jxb/erh048

Gliwicka M, Nowak K, Balazadeh S, Mueller-Roeber B, Gaj MD (2013) Extensive modulation of the transcription factor transcriptome during somatic embryogenesis in Arabidopsis thaliana. PLoS One. doi:10.1371/journal.pone.0069261

Gomes HT, Bartos PMC, Silva CO, Velho do Amaral LI, Scherwinski-Pereira JE (2014) Comparative biochemical profiling during the stages of acquisition and development of somatic embryogenesis in African oil palm (Elaeis guineensis Jacq.). Plant Growth Regul 74:199-208. doi:10.1007/s10725-014-9901-4

Grieb B, Schafer F, Imani J, Mashayekhi KN, Arnholdt-Schmitt B, Neumann KH (1997) Changes in soluble proteins and phytohormone concentrations of cultured carrot petiole explants during induction of somatic embryogenesis (Daucus carota L.). J Appl Bot Food Qual 71:94-103
Hura T, Dziurka M, Hura K, Ostrowska A, Dziurka K (2016) Different allocation of carbohydrates and phenolics in dehydrated leaves of triticale. J Plant Physiol 202:1-9. doi:10.1016/j. jplph.2016.06.018

Ivanova A, Velcheva M, Denchev P, Atanassov A, Van Onckelen HA (1994) Endogenous hormone levels during direct somatic embryogenesis in Medicago falcata. Physiol Plant 92:85-89. doi:10.1111/j.1399-3054.1994.tb06658.x

Iwase A, Mitsuda N, Koyama T, Hiratsu K, Kojima M, Arai T, Inoue Y, Seki M, Sakakibara H, Sugimoto K, Ohme-Takagi M (2011) The AP2/ERF transcription factor WIND1 controls cell dedifferentiation in Arabidopsis. Curr Biol 21:508-514. doi:10.1016/j.cub.2011.02.020

Janeczko A, Biesaga-Kościelniak J, Oklest'kova J, Filek M, Dziurka M, Szarek-Łukaszewska G, Kościelniak J (2010) Role of 24-epibrassinolide in wheat production: physiological effects and uptake. J Agron Crop Sci 196:311-321. doi:10.1111/j.1439-037X.2009.00413.x

Jiménez VM, Bangerth F (2000) Relationship between endogenous hormone levels of grapevine callus cultures and their morphogenetic behaviour. Vitis 39:151-157.

Jiménez VM, Bangerth F (2001a) Hormonal status of maize initial explants and of the embryogenic and non-embryogenic callus cultures derived from them as related to morphogenesis in vitro. Plant Sci 160:247-257. doi:10.1016/ S0168-9452(00)00382-4

Jiménez VM, Bangerth F (2001b) Endogenous hormone levels in explants and in embryogenic and non-embryogenic cultures of carrot. Physiol Plant 111:389-395. doi:10.1034/j.1399-3054.2001.1110317.x

Jiménez VM, Guevara E, Herrera J, Bangerth F (2005) Evolution of endogenous hormone concentration in embryogenic cultures of carrot during early expression of somatic embryogenesis. Plant Cell Rep 23:567-572. doi:10.1007/s00299-004-0869-9

Kikuchi A, Sanuki N, Higashi K, Koshiba T, Kamada H (2006) Abscisic acid and stress treatment are essential for the acquisition of embryogenic competence by carrot somatic cells. Planta 223:637-645. doi:10.1007/s00425-005-0114-y

León J, Rojo E, Sánchez-Serrano JJ (2001) Wound signalling in plants. J Exp Bot 52:1-9. doi:10.1093/jexbot/52.354.1

Limanton-Grevet A, Sotta B, Brown S, Jullien M (2000) Analysis of habituated embryogenic lines in Asparagus officinalis L.: growth characteristics, hormone content and ploidy level of calli and regenerated plants. Plant Sci 160:15-26. doi:10.1016/ S0168-9452(00)00356-3

Liu ZH, Liu H-Y, Wang H-Y (1996) Effect of light on indole-3-acetic acid in soybean hypocotyls. Bot Bull Acad Sin 37:113-119.

Macháčková I, Eder J, Motyka V, Hanuš J, Krekule J (1996) Photoperiodic control of cytokinin transport and metabolism in Chenopodium rubrum. Physiol Plant 98:564-570

May RA, Trigiano RN (1991) Somatic embryogenesis and plant regeneration from leaves of Dendranthema grandiflora. J Am Soc Hortic Sci 116:366-371

Mikuła A, Pożoga M, Grzyb M, Rybczyński JJ (2015a) An unique system of somatic embryogenesis in the tree fern Cyathea delgadii Sternb.: the importance of explant type, and physical and chemical factors. Plant Cell, Tissue Organ Cult 123:467-478. doi:10.1007/s11240-015-0850-z

Mikuła A, Pożoga M, Tomiczak K, Rybczyński JJ (2015b) Somatic embryogenesis in ferns: a new experimental system. Plant Cell Rep 34:783-794. doi:10.1007/s00299-015-1741-9

Mohr H, Schopfer P (1995) Physiology of hormone action. In: Mohr H, Schopfer P (eds) Plant Physiology. Springer, Berlin, pp 383-408

Murashige T, Skoog F (1962) A revised medium for rapid growth and bioassays with tobacco tissue cultures. Physiol Plant 15:473-497 
Pacholczak A, Szydło W, Łukaszewska A (2005) The effect of etiolation and shading of stock plants on rhizogenesis in stem cuttings of Cotinus coggygria. Acta. Physiol Plant 27:417-428. doi:10.1007/s11738-005-0046-y

Pérez-Jiménez M, Cantero-Navarro E, Acosta M, Cos-Terrer J (2013) Relationships between endogenous hormonal content and direct somatic embryogenesis in Prunus persica L. Batsch cotyledons. Plant Growth Regul 71:219-224. doi:10.1007/ s10725-013-9822-7

Pintos B, Martín JP, Centeno ML, Villalobos N, Guerra H, Martín L (2002) Endogenous cytokinin levels in embryogenic and nonembryogenic calli of Medicago arborea L. Plant Sci 163:955960. doi:10.1016/S0168-9452(02)00244-3

Raman N, Elumalai S (1996) Presence of cytokinin in the root nodules of Casuarina equisetifolia. Indian J Exp Biol 34:577-579

Seo M, Koshiba T (2002) Complex regulation of ABA biosynthesis in plants. Trends Plant Sci 7:41-48. doi:10.1016/ S1360-1385(01)02187-2

Smith DL, Krikorian AD (1989) Release of somatic embryogenic potential from excised zygotic embryos of carrot and maintenance of proembryonic cultures in hormone-free medium. Am J Bot 76:1832-1843

Štefančič M, Štampar F, Veberič R, Osterc G (2007) The levels of IAA, IAAsp and some phenolics in cherry rootstock "GiSelA 5" leafy cuttings pretreated with IAA and IBA. Sci Hortic (Amsterdam) 112:399-405. doi:10.1016/j.scienta.2007.01.004

Stone SL, Braybrook SA, Paula SL, Kwong LW, Meuser J, Pelletier J, Hsieh T-F, Fischer RL, Goldberg RB, Harada JJ (2008) Arabidopsis LEAFY COTYLEDON2 induces maturation traits and auxin activity: Implications for somatic embryogenesis. PNAS 105:3151-3156

Suzuki RM, Kerbauy GB (2006) Effects of light and ethylene on endogenous hormones and development of Catasetum fimbriatum (Orchidaceae). Brazilian. J Plant Physiol 18:359-365. doi:10.1590/S1677-04202006000300002

Symons GM, Reid JB (2003) Interactions between light and plant hormones during de-etiolation. J Plant Growth Regul 22:3-14. doi:10.1007/s00344-003-0017-8
Takamura T, Tanaka M (1996) Somatic embryogenesis from the etiolated petiole of cyclamen (Cyclamen persicum Mill.). Plant Tissue Cult Lett 13:43-48.

Thornburg RW, Li X (1991) Wounding Nicotiana tabacum leaves causes a decline in endogenous indole-3-acetic acid. Plant Physiol 96:802-805. doi:10.1104/pp.96.3.802

Volmaro C, Pontin M, Luna V, Baraldi R, Bottini R (1998) Blue light control of hypocotyl elongation in etiolated seedlings of Lactuca sativa (L.) cv. Grand Rapids related to exogenous growth regulators and endogenous IAA, GA3 and abscisic acid. Plant Growth Regul 26:165-173

Wenck AR, Conger BV, Trigiano RN, Sams CE (1988) Inhibition of somatic embryogenesis in Orchardgrass by endogenous cytokinins. Plant Physiol 88:990-992

Wójcikowska B, Gaj MD (2015) LEAFY COTYLEDON2-mediated control of the endogenous hormone content: implications for the induction of somatic embryogenesis in Arabidopsis. Plant Cell, Tissue Organ Cult 121:255-258. doi:10.1007/ s11240-014-0689-8

Yang X, Zhang X, Yuan D, Jin F, Zhang Y, Xu J (2012) Transcript profiling reveals complex auxin signalling pathway and transcription regulation involved in dedifferentiation and redifferentiation during somatic embryogenesis in cotton. BMC Plant Biol. doi:10.1186/1471-2229-12-110

Zheng Q, Zheng Y, Perry SE (2013) AGAMOUS-Like15 promotes somatic embryogenesis in Arabidopsis and soybean in part by the control of ethylene biosynthesis and response. Plant Physiol 161:2113-2127. doi:10.1104/pp.113.216275

Żur I, Dubas E, Krzewska M, Waligórski P, Dziurka M, Janowiak F (2015) Hormonal requirements for effective induction of microspore embryogenesis in triticale $(\times$ Triticosecale Wittm.) anther cultures. Plant Cell Rep 34:47-62. doi:10.1007/ s00299-014-1686-4 